RAIRO Operations Research

RAIRO Oper. Res. 35 (2001) 85-106

\title{
MULTIPLE ROUTING STRATEGIES IN A LABELLED NETWORK
}

\author{
J. Maublanc ${ }^{1}$, D. Peyrton ${ }^{1}$ And A. Quilliot ${ }^{2}$ \\ Communicated by P. Chrétienne
}

\begin{abstract}
We present here models and algorithms for the construction of efficient path systems, robust to possible variations of the characteristics of the network. We propose some interpretations of these models and proceed to numerical experimentations of the related algorithms. We conclude with a discussion of the way those concepts may be applied to the design of a Public Transportation System.
\end{abstract}

Keywords: Shortest paths, network design, routing.

\section{INTRODUCTION}

Shortest path problems are among the combinatorial optimization problems which have been the most widely studied during the last thirty years. Applications to stock management, planning, routing and network design have first led to very well-known algorithms designed for explicitly defined networks: Bellman algorithms for acyclic networks [8,17], Dijkstra algorithm for positive networks [2,3], Dantzig algorithm for the general case... [9,11,19]. Applications related to robotics and strategic games have next motivated several adaptations of these methods: $\mathrm{A}^{*}$ and $\mathrm{B}^{*}$ algorithms for very large scale state networks, stochastic models for the case when the effects of some actions or transitions cannot be predicted in a deterministic way $[1,10,14,16]$.

It may occur that we cannot expect to get a complete knowledge of the future state of the network at the time when we are required to handle the path search

Received January, 1996. Accepted November, 2000.

1 CERTU, Lyon, France.

2 ISIMA, Campus des Cézeaux, Université Blaise Pascal, BP. 125, 63173 Aubière, France.

(C) EDP Sciences 2001 
problem. In such a case, we need to look for several paths, which at the same time are the most efficient possible and also are pairwise independent with respect to the possible future configurations of the network. For instance, routing messages or goods through some telecommunication or transportation network or through some multiprocessor MIMD architecture is usually managed in two steps: the first one consists in prealably computing some convenient candidate paths, and the second one consists, every time the routing process is launched, in picking up $[5-7,13,21]$, while taking into account the current state of the network, the path which seems the most efficient at this time.

Then the underlying problem consists in searching, for a given origin/destination pair $(\mathrm{o}, \mathrm{d})$, some path family, which is made with paths from o to d, independent in the sense that their performances under the possible variations of the state of the network are not too narrowly correlated, and which defines at the same time some kind of shortest path family.

It is with this problem which we are going to deal here. We shall first propose some general model, aimed at providing some formalization of the above notion of independence, together with some examples and resolution algorithms. We will discuss the complexity of these algorithms and conclude by presenting numerical experimentations.

\section{A GENERAL MODEL}

\subsection{Preliminaries}

Let us consider some network $G=(X, E)$. For any arc $e$ in $G$, we denote by $o(e)$ the origin of $e$ and by $s(e)$ the extremity of $e$.

A path $\gamma$ in $G$ is a finite sequence $\gamma=e_{1} \ldots e_{n}$ of arcs in $E$ such that for any $i=1 \ldots n-1, o\left(e_{i+1}\right)=s\left(e_{i}\right)$. The vertex $o\left(e_{1}\right)$ is called the origin of $\gamma$ and is denoted by or $(\gamma)$; the vertex $s\left(e_{n}\right)$ is called the extremity of $\gamma$ and is denoted by $\operatorname{ext}(\gamma)$. Such a path $\gamma$ is said to be elementary if the vertices $o\left(e_{i}\right), i=1 \ldots n$, and $s\left(e_{n}\right)$, are pairwise distinct.

The vertex sequence $o\left(e_{1}\right), \ldots, o\left(e_{n}\right), s\left(e_{n}\right)$, is called the support of the path $\gamma$ and is denoted by $\operatorname{Supp}(\gamma)$.

In case no two arcs of $G$ connect the same pair of vertices, we consider that $\gamma$ is completely defined by its support.

If $d$ is a length function which to any arc $e$ in $E$ makes correspond some real number $d(e)$, then we set:

$$
d^{*}(\gamma)=\sum_{i=1 \ldots n} d\left(e_{i}\right)=d \text {-length of } \gamma
$$

If $x$ and $y$ are two vertices in the support of $\gamma$, such that $x$ is located before $y$ on $\operatorname{Supp}(\gamma)$, then we denote by $\gamma_{x, y}$ the subpath of $\gamma$ whose origin is $x$ and whose extremity is $y$, and we call it the restriction of $\gamma$ from $x$ to $y$. 
We denote by $\mathbf{P}(\mathbf{G})$ the set of all the elementary paths of $G$ and by $\mathbf{P}^{*}(\mathbf{G})$ the set of all the paths of $G$.

We denote by Nil the empty path, which may be viewed as connecting any vertex to itself.

If $\gamma$ and $\gamma^{\prime}$ are two paths such that $\operatorname{or}\left(\gamma^{\prime}\right)=\operatorname{ext}(\gamma)$, then we denote by $\gamma+\gamma^{\prime}$ the concatenation of $\gamma$ and $\gamma^{\prime}$.

For any pair $x, y$ of vertices of $G$, we denote by $P(G)_{x, y}$ the set of all elementary paths whose origin is $x$ and whose extremity is $y$.

\subsection{Notion of Strategic triple}

The crucial notion behind our problem is the notion of independence. We want to express the fact that several paths are efficient, in the sense that they allow a fast connection between two given vertices, while being independent with relation to the evolution of some set of state parameters.

Practically, two paths will be considered as independent, if their underlying strategies are not the same and if their performance are not tied to each other in a logical way. When talking about strategy, we may think for instance into some traveler which moves from one area to another: its underlying strategy is basically defined by the sequence of transportation modes that he uses. More generally, the arcs of the network $G=(X, E)$ are in most of the cases endowed with some kind of symbolic or numerical information, and the notions of equivalence or domination between strategies must express themselves through some equivalence or partial ordering relations on the word set defined by all the possible concatenations of those informations.

In order to cast these intuitions into a general model, let us consider some network $G=(X, E)$.

We call Strategic Triple defined on $G$, any triple $(R, O, L)$ where:

- $R$ is an equivalence relation defined on the path set $P(G)^{*}$;

- $O$ is a partial order relation defined on $P(G)^{*} ;(O$ may be read "more efficient than");

- $L$ is a subset of $P(G)^{*}$;

which is such that:

(H1) $O$ and $R$ are compatible, which means that if $\gamma, \gamma^{\prime}$ and $\gamma^{\prime \prime}$ in $P(G)^{*}$ are such that:

$-\gamma^{\prime} R \gamma^{\prime \prime}$

- $\gamma O \gamma^{\prime}\left(\gamma^{\prime} O \gamma\right)$

then we also have: $\gamma O \gamma^{\prime \prime}\left(\gamma^{\prime \prime} O \gamma\right)$.

(H2) For any $\gamma$ in $P(G)^{*}$, Nil $R \gamma$ or Nil $O \gamma(\operatorname{Nil}(O \vee R) \gamma)$.

(H3) $R$ and $O$ are stable by right side concatenation, which means that if $\gamma$, $\gamma^{\prime}$ and $\gamma^{\prime \prime}$ in $P(G)^{*}$ satisfy:

- $\gamma^{\prime} R(O) \gamma^{\prime \prime}$

$-\operatorname{or}(\gamma)=\operatorname{ext}\left(\gamma^{\prime}\right)=\operatorname{ext}\left(\gamma^{\prime \prime}\right)$;

then we also have: $\left(\gamma^{\prime}+\gamma\right) R(O)\left(\gamma^{\prime \prime}+\gamma\right)$. 
(H4) There exists a projection operator $\Pi$ from $P(G)^{*}$ to $L$ which is such that:

- for any $\gamma$ in $L, \Pi(\gamma)=\gamma$;

- for any $\gamma$ in $P(G)^{*}, \operatorname{or}(\Pi(\gamma))=\operatorname{or}(\gamma)$ and $\operatorname{ext}(\Pi(\gamma))=\operatorname{ext}(\gamma)$;

- $\Pi$ is an homomorphism for both relations $R$ and $O \vee R$ (it conserves both relations);

- if $\gamma$ is in $L$, and if $u$ is an $\operatorname{arc}$ of $G$, such that $\operatorname{ext}(\gamma)=o(u)$, then there exists some vertex $x$ in the support of $\gamma$ such that the arc $[x, s(u)]$ exists and that $\Pi(\gamma+\{u\})=\gamma_{\text {or }(\gamma), x}+\{[x, s(u)]\}$.

(H5) Any subpath of some path $\gamma$ in $L$ is also in $L$.

If $d$ is a positive length function defined on the arc set of $G$, we say that $d$ is compatible with the strategic triple $(R, O, L)$ if for any path $\gamma$ in $P(G)^{*}$, we have: $d^{*}(\Pi(\gamma)) \leq d^{*}(\gamma)$

Comments. The equivalence relation $R$ formalizes here the notion of strategy. A strategy is an equivalence class for $R$. We call Strategy Set associated with $G=(X, E)$ and with the strategic triple $(R, O, L)$ the quotient set $P(G)^{*} / R$, and we denote it by $\operatorname{ST}(G, R)$.

Axiom (H1) means that the partial ordering $O$ defines a partial ordering $O^{*}$ on the Strategy Set $\operatorname{ST}(G, R)$.

Axiom (H2) and (H3) mean that any subpath of a given path $\gamma$ is usually less costly or more fiable than $\gamma$.

The subset $L$ of $P(G)^{*}$ expresses the fact that the concept of strategy may be restrained by some syntactical constraints. If for instance we think into a strategy as being some sequence of transportation modes, we will only consider alternated mode sequences, that means sequences which never contain 2 identical consecutive symbols. In such a case, Axiom (H4) ensures that to any path of $G$ will correspond some strategy.

\subsection{Minimal Independent PATH FAMilies}

So, let $G=(X, E)$ be some network, let $(R, O, L)$ be some strategic triple on $G$ and let $x, y$ be two vertices in $G$.

Two pathes $\gamma$ and $\gamma^{\prime}$ in $P(G)^{*}$ are said to be $R$-equivalent iff $\gamma R \gamma^{\prime}$; they are said to be $R$-independent iff they are not $R$-equivalent.

We say that $\gamma O$-dominate $\gamma^{\prime}$ iff $\gamma O \gamma^{\prime}$; we say that $\gamma$ and $\gamma^{\prime}$ are strongly $(O, R)$-independent iff they are $R$-independent and if none of both $O$-dominates the other.

A family $\Lambda=\left(\gamma_{1} \ldots \gamma_{k}\right)$ of pathes of $P(G)_{x, y}$ will be called a $k$ minimal independent path family from $x$ to $y$ associated with the strategic triple $(R, O, L)$ iff:

- the pathes in $\Lambda$ are all in $L$ and are pairwise $R$-independent;

- if $i$ in $\{1 \ldots k\}$, and $\gamma$ in $P(G)_{x, y}$ are such that $\gamma O \gamma_{i}$, then there exists $j<i$ such that $\gamma R \gamma_{j}$.

Let us suppose now that $G$ is endowed with some real valued length function $d$, defined on its arc set $E$ and compatible with $(R, O, L)$. A family $\Lambda=\left(\gamma_{1} \ldots \gamma_{k}\right)$ 
of pathes of $P(G)_{x, y}$ will be said to be a $k$ minimal strongly independent path family from $x$ to $y$ associated with the strategic triple $(R, O, L)$ and with the length function $d$, iff:

- the pathes in $\Lambda$ are all in $L$ and are pairwise strongly $(O, R)$-independent;

- if $i=1 \ldots k$, and $\gamma$ in $P(G)_{x, y}$ are such that $d^{*}(\gamma)<d^{*}\left(\gamma_{i}\right)$, then there exists $j \leq i$ such that $\gamma_{j} O \gamma$ or that $\gamma_{j} R \gamma$ and $d^{*}\left(\gamma_{j}\right)<d^{*}(\gamma)$.

One may raise two problems:

Problem 1. Given some network $G=(X, E)$, some strategic triple $(R, O, L)$, some pair $x, y$ of vertices of $G$, and some integer $k$, find an associated $k$ minimal independent path family from $x$ to $y$.

Problem 2. Given some network $G=(X, E)$, some strategic triple $(R, O, L)$, some pair $x, y$ of vertices of $G$, some length function $d$ defined on $E$ and compatible with $(R, O, L)$, and some integer $k$, find an associated $k$ minimal strongly independent path family from $x$ to $y$.

Remark. Both above concepts aim at providing us with a formal framework for the search of a family of independent efficient paths in a network. One may notice, taking into account axioms (H2) and (H3) of item II.2, that restricting ourselves to elementary paths from $x$ to $y$ in the above definitions does not really means the introduction of any additional constraint. Besides, the study of some examples will allow us to notice that any solution of Problem 2 is also a solution of Problem 1 and that solving Problem 2 produces more information than solving Problem 1. For this, most of our work will focus on the study of Problem 2.

\section{EXAMPLES AND COMMENTS}

\subsection{A simple mathematical example}

Let us consider some network $G=(X, E)$, some alphabet $A$, and let us suppose that any $\operatorname{arc}$ in $E$ is endowed with some symbol in $A$, in such a way that:

- if the $\operatorname{arcs}[x, y]$ and $[y, z]$ exist and are both labelled with the same symbol $u$, then the arc $[x, z]$ exists and is also labelled with $u$;

Then to any path $\gamma$ in $G$ corresponds some word $m(\gamma)$ in the word set $M(A)$ defined on $A$.

We obtain a strategic triple $(R, O, L)$ by setting:

- $\gamma R \gamma^{\prime}$ iff the words $m(\gamma)$ and $m\left(\gamma^{\prime}\right)$ are the same;

- $\gamma O \gamma^{\prime}$ iff the word $m(\gamma)$ is a subword of the word $m\left(\gamma^{\prime}\right)$;

and by defining $L$ as being the set of all alternated pathes, i.e., all pathes which do not contain two consecutive arcs labelled with the same symbol.

In case some positive length function $d$ is defined on $E$, we see that $d$ is compatible with $(R, O, L)$ iff for any pair of $\operatorname{arcs}[x, y]$ and $[y, z]$ endowed with the same label, we have $d([x, z]) \leq d([x, y])+d([y, z])$. 
b, 6

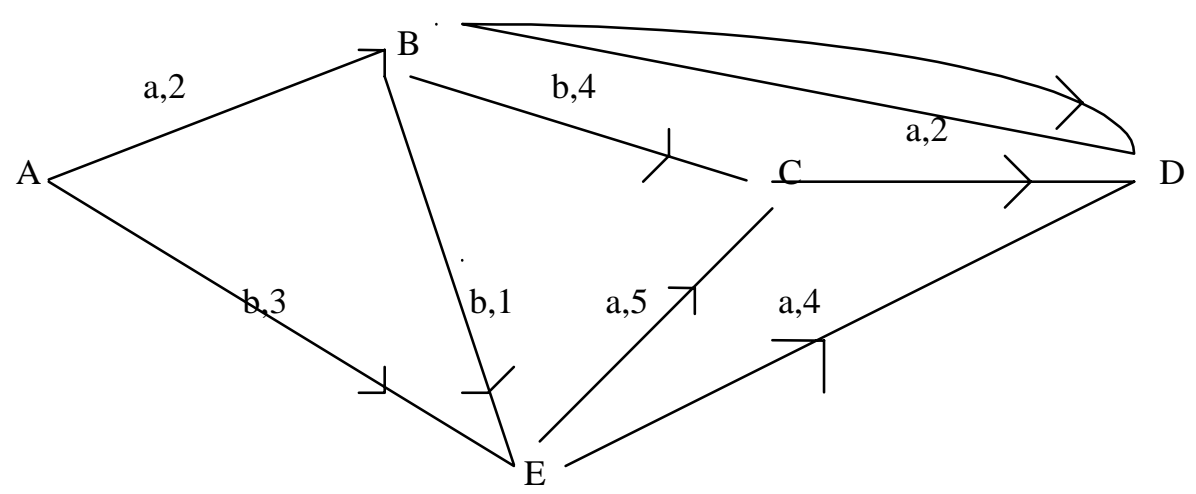

We may consider the following small example:

Vertex set $X=\{A, B, C, D, E\}$;

Arc set $E=([A, B],[A, E],[B, E],[B, C],[E, C],[C, D],[E, D],[B, D])$, those arcs respectively endowed with the labels $a, b, b, b, a, a, a, b$ and with the lengthes 2 , $3,1,4,5,2,4,6$.

Paths $(A, B, C, D)$ and $(A, B, E, D)$ are here $R$-equivalent, and are both $O$-dominated by path $(A, E, D)$.

We also see here how the projection operator $\Pi$ works: to the path with support $(A, E, C, D)$ it makes correspond the path with support $(A, E, D)$.

If $k=2$, and if $x=A, y=D$, we see that Problem 1 admits exactly two symmetric solutions:

- $\gamma_{1}=(A, E, D)$ and $\gamma_{2}=(A, B, D)$;

- $\gamma_{1}=(A, B, D), \gamma_{2}=(A, E, D)$.

Problem 2 admits exactly one solution: $\gamma_{1}=(A, E, D)$ and $\gamma_{2}=(A, B, D)$.

\subsection{An EXAmple RELATED to tRAnSPORTATiOn Systems}

Let us now look at an example which will be used several times during the rest of the paper.

We consider a network $G=(X, E), 2$ vertices $x_{0}$ and $y_{0}$ in $X$, and a set $U$ of symbolic variables which provides some labelling of the $\operatorname{arcs}$ of $G$. $G$ is supposed to represent some transportation infrastructure and any symbolic identifier $u$ in $U$ may be considered as the identifier of some speed variable. More precisely, we suppose that if an $\operatorname{arc} e=[x, y]$ is given and if the value of $U(e)$ is known, then the time required to go from $x$ to $y$ is given by an expression:

$$
t(e, U)=\alpha(e)+\lambda(e) \cdot U(e)
$$

where $\alpha(e)$ and $\lambda(e)$ are positive coefficients associated with $e$. 
That means that if $\gamma$ is some path, made with consecutive $\operatorname{arcs} e_{1} \ldots e_{n}$, the time $\sum_{i=1 \ldots n} t\left(e_{i}, w\right)$ required to run along $\gamma$ appears as a formal $U$-expression:

$$
\Lambda^{*}(\gamma)=\Delta(\gamma)+\sum_{u \operatorname{in} U} \Lambda(u, \gamma) \cdot u
$$

which is linear in the decision vector $U$.

It may occur that we need to handle the shortest path problem between $x_{0}$ and $y_{0}$ without knowing what will be the exact value of the vector $U$, at the time when the run from $x_{0}$ to $y_{0}$ will effectively take place. Such a situation may come for instance from the fact that the effective running decision needs to be a real time decision, which makes impossible full information gathering and shortest path computing, or also from the fact that the shortest path handling process may be part of a global design process aimed the determination of the value of $U$.

In such a case, we try to get, not a single optimal path, but a whole path family likely to produce, at the time the running decision will be taken, and whatever be then the exact value of $w$, at least one good strategy.

In a natural way, we consider here that 2 pathes $\gamma_{1}$ and $\gamma_{2}$ are $R$-equivalent if both formal expressions $\Lambda^{*}\left(\gamma_{1}\right)$ and $\Lambda^{*}\left(\gamma_{2}\right)$ are the same and that path $\gamma_{1} O$ dominates $\gamma_{2}$ if $\Lambda^{*}\left(\gamma_{1}\right)$ is never larger that $\Lambda^{*}\left(\gamma_{2}\right)$, whatever be the feasible value of the vector $U$. We define $L$ as being the set $P^{*}(G)$.

Let us consider for instance the following example:

$X=\{(A, B, C, D, E, F, G)\}$

$U=\{u, v\}$

$E=\{[A, B],[A, C],[B, E],[E, F],[C, D],[D, F],[A, G],[G, F],[B, D]\}$, those arcs $e$ being respectively endowed by the following linear affine expressions $t(e, U): u, v+1, v+2,2 v, u+2,3, u+4,2 v+3, v+3$.

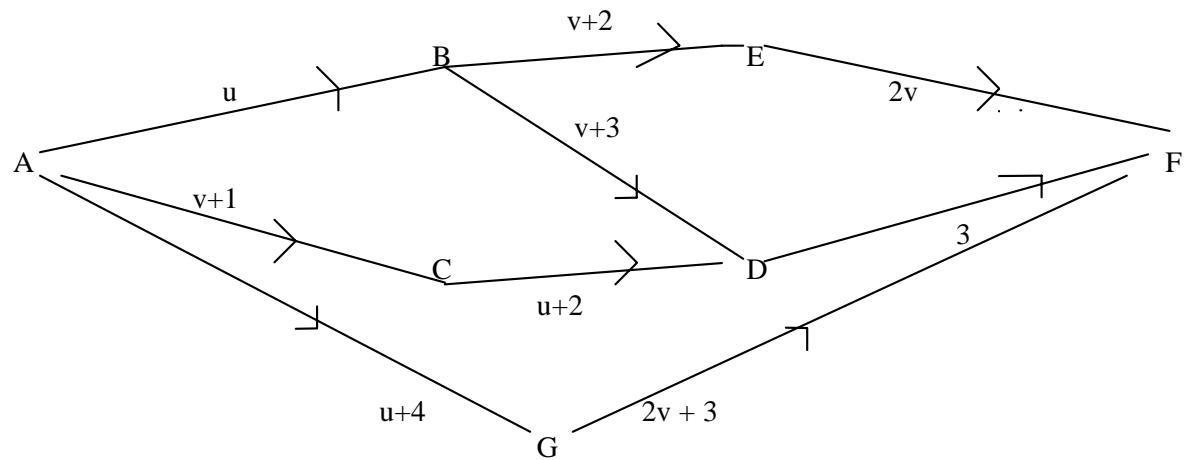

We see that paths $(A, B, D, F)$ and $(A, C, D, F)$ are $R$-equivalent and that they both $O$-dominate path $(A, G, F)$.

Let us suppose $k=2, x_{0}=A, y_{0}=F$. 
Then, Problem 1 admits here 3 solutions, modulo $R$-equivalence:

First solution: $\gamma_{1}=\operatorname{path}(A, B, D, F)$ and $\gamma_{2}=$ path $(A, G, F)$;

Second solution: $\gamma_{1}=$ path $(A, B, D, F)$ and $\gamma_{2}=$ path $(A, B, E, F)$;

Third solution: $\gamma_{2}=$ path $(A, B, D, F)$ and $\gamma_{1}=$ path $(A, B, E, F)$.

Let us suppose now that the mean value of $u$ is 5 and that the mean value of $v$ is 0.5. Thus, we may associate with any arc $e$ in $E$, some quantity $d(e)$ which is the mean value of $t(e, U)$.

Then we see that the length function $d$ defined this way is compatible with the strategic triple $(R, O, L)$ and that Problem 2 has exactly one solution (modulo $R$-equivalence):

$$
\gamma_{1}=\operatorname{path}(A, B, E, F) ; \gamma_{2}=\text { path }(A, B, D, F)
$$

We also see that if $k=3$, then Problem 2 does not admit any solution.

We see on this example that Problem $\mathbf{2}$ is much more constraining than Problem 1, and that answering it is going to provide us with much more information about what will be a good strategy for moving from some origin to some destination, according to the variations of the state of our system.

\subsection{An EXAmple RELATED tO RELiability}

Once again we consider some network $G=(X, E)$, and some length function $d$ defined on the arc set $E$ of $G$.

We consider also a set $U$ of independent $\{0,1\}$ random variables and we suppose that at any instant, every variable in $U$ commands the access to several arcs of $E$.

Thus to any path $\gamma$ in $G$ corresponds a $U$-monomial $m(\gamma)$ which provides the probability that $\gamma$ may be used at a given time, and which is such that the degree in $m(\gamma)$ of any variable $u$ in $U$ is 0 or 1 .

At any time, we may be required to connect two given vertices $x_{0}$ and $y_{0}$ with some shortest valid path. Since performing the associated computing under real time constraints may eventually induce some trouble, we prealably extract some path family, which are the shortest possible for the length function $d$, and which are independent with regard to the way the variables of $U$ behave.

In order to do it, we set:

- $L=$ all the paths in $G$;

$-\gamma R \gamma^{\prime}$ iff $m(\gamma)=m\left(\gamma^{\prime}\right)$

- $\gamma O \gamma^{\prime}$ iff $m(\gamma) \geq m\left(\gamma^{\prime}\right)$ whatever be the values taken by the variables of $U$.

Thus, we are typically in the situation of looking (Problem 2 ) for some $k$ minimal strongly independent path family from xo to yo associated with the strategic triple $(R, O, L)$ and with the length function $d, k$ being some conveniently chosen integer.

Once this family has been computed, we handle our problem by picking up inside this family, every time it is necessary, the shortest currently valid path. 


\section{THE MAIN ALGORITHMS}

In all this section, we are going to consider some network $G=(X, E)$, some origin/destination pair $\left(x_{0}, y_{0}\right)$, some strategic triple $(R, O, L)$, some positive length function $d$ defined on the arc set $E$ and compatible with $(R, O, L)$, and some integer $k$.

We first do some few remarks:

- Because of its compatibility with the equivalence relation $R$, the partial ordering $O$ turns itself into a partial ordering $O^{*}$ defined on the Strategy Set $\operatorname{ST}(G, R)=P(G)^{*} / R$. A solution of Problem 1 corresponds to some subset $S$ of $\operatorname{ST}(G, R)$, such that:

- if $s$ is in $S$, then any $s^{\prime}$ such that $s^{\prime} O^{*} s$ is also in $S$.

Clearly, such a subset does not need to be unique, as we noticed in the previous section. A solution of Problem 2 corresponds to some subset $S$ of $\operatorname{ST}(G, R)$ which is made only with elements which are minimal for the partial ordering $O^{*}$.

Thus, any solution of Problem 2 is also a solution of Problem 1.

- Let $\operatorname{MIN}(G, R)$ be the subset of $\operatorname{ST}(G, R)$ defined by all the elements which are minimal for $O^{*}$. For any strategy $s$ in $\operatorname{ST}(G, R)$, we may define the following quantity:

$H(s)=\operatorname{Inf} d^{*}(\gamma)$, for all the paths $\gamma$ being in the $R$-equivalence associated with $s$.

Thus, solving Problem 2 simply means finding some subset $S$ of $\operatorname{MIN}(G, R)$ which yields the $k$ smallest possible values $H(s), s$ in $\operatorname{MIN}(G, R)$.

It follows that, if it exists, any solution $\gamma_{1} \ldots \gamma_{k}$ of Problem $\mathbf{2}$ is unique in the sense that:

- the sequence of values $d^{*}\left(\gamma_{i}\right), i=1 \ldots k$, is completely determined;

- for any path $\gamma$ from $x_{0}$ to $y_{0}$ such that $d^{*}(\gamma)<\operatorname{Sup} d^{*}\left(\gamma_{i}\right), i=1 \ldots k$, and such that the equivalence class of $\gamma$ is in $\operatorname{MIN}\left(P(G)^{*} / R\right)$, there must exist exactly one value $i$ such that $\gamma$ is $R$-equivalent to $\gamma_{i}$.

These remarks justify the fact that most of the next sections will be devoted to the study of Problem 2.

\subsection{Algorithm STRATPATH2 For the Problem 2}

In order to present this algorithm, we introduce some additional notations:

- Relation $O_{<}$: if $\gamma$ and $\gamma^{\prime}$ in $P(G)^{*}$ are such that either $\gamma O \gamma^{\prime}$ or $\left[\left(\gamma R \gamma^{\prime}\right)\right.$ and $\left.d^{*}(\gamma)<d^{*}\left(\gamma^{\prime}\right)\right]$ then we set $\gamma O_{<} \gamma^{\prime}$;

- Relation $R_{=}$: if $\gamma$ and $\gamma^{\prime}$ in $P(G)^{*}$ are such that $\gamma R \gamma^{\prime}$ and $d^{*}(\gamma)=d^{*}\left(\gamma^{\prime}\right)$ then we set $\gamma R=\gamma^{\prime}$.

Algorithm STRATPATH2 is designed as an extension of Dijkstra Algorithm for the shortest path problem in positive networks. It is structured into one main 
loop, in such a way that at every entry into this main loop the situation is the following one:

- For some pairs $(x, i), x$ in $X, i$ in $N$, an elementary path $\Gamma(x, i)$ from $x_{0}$ to $x$ has been computed together with some length $\Pi(x, i)$. For any such a pair $(x, i)$ with this property, and for any index $j$ in $1 \ldots i$, the path $\Gamma(x, j)$ and the length $\Pi(x, j)$ also exist and the paths $\Gamma(x, j), j=1 \ldots i$, are strongly $(O, R)$-independent. For any given vertex $x$, the sequence of the existing lengthes $\Pi(x, i)$ is increasing. Besides, some of these pairs $(x, i)$ have been marked, and if some pair $(x, i)$ is marked, then also is any pair $(x, j)$ with $j$ in $1 \ldots i$.

Then the following actions are performed:

- Some non marked pair $(x, i)$ is selected, in such a way that $\Gamma(x, i)$ is minimal for the relation $O_{<}$among the paths associated with non marked pairs; this pair is called the pivot pair and becomes marked.

- For any arc $[x, y]$ in $E$, such that the path $\Gamma(x, i)+\{[x, y]\}$ is in $L$ and such that no path $\Gamma(y, j)$ satisfies:

$$
\Gamma(y, j) O_{<}(\Gamma(x, i)+\{[x, y]\})
$$

or

$$
\left(\Gamma(y, j) R_{=}(\Gamma(x, i)+\{[x, y]\})\right.
$$

then we clean up the sequence $(\Gamma(y, u), \Pi(y, u))$ for $u \geq 1$ in such a way that:

- it contains the pair $\left(\Gamma(x, i)+\{[x, y]\}, d^{*}(\Gamma(x, i)+\{[x, y]\})\right)$;

- it is ordered through increasing values $\Pi(y, u)$;

- the paths $\Gamma(y, u), u \geq 1$, which appear in this finite sequence remain strongly $(O, R)$-independent.

The process stops when the pair $\left(y_{0}, k\right)$ is marked or when no pivot pair $(x, i)$ may be selected.

The algorithm STRAT-PATH2 may be formally described as follows:

\section{Algorithm STRAT-PATH2}

Input: A network $G=(X, E)$, an origin/destination pair $\left(x_{0}, y_{0}\right)$, a strategic triple $\overline{(R, O}, L)$, a length function $d$ defined on $E$ and compatible with $(R, O, L)$ and an integer $k$.

Output: Failure or a $k$ minimal strongly independent path family from $x_{0}$ to $y_{0}$ associated with $(R, O, L)$ and $d$;

$\Gamma\left(x_{0}, 0\right):=$ Trivial path reduced to the vertex $x_{0}$;

Not Stop; No pair $(x, i)$ is marked;

\section{While Not Stop do}

Set (Pivot, Index-Pivot): = some non marked pair $(x, i)$ such that $\Gamma(x, i)$ exists and is $O_{<- \text {minimal; }}$

If (Pivot, Index-Pivot) does not exist then Stop (failure)

else 
if (Pivot, Index-Pivot) $=\left(y_{0}, k\right)$ then Stop $\left(\right.$ Success: the $\Gamma\left(y_{0}, i\right)$, $i=1 \ldots k$, are the result)

else

Mark (Pivot, Index-Pivot);

For any arc $[x, y]$ such that $\Gamma(x, i)+\{[x, y]\}$ is in $L$ and such that no $\operatorname{path} \Gamma(y, j)$ satisfies:

$$
\begin{aligned}
& \Gamma(y, j) O_{<}(\Gamma(x, i)+\{[x, y]\}) \\
& \text { or } \\
& \left(\Gamma(y, j) R_{=}(\Gamma(x, i)+\{[x, y]\})\right.
\end{aligned}
$$$$
\text { do }
$$

Set $\Gamma:=\Gamma(x, i)+\{[x, y]\}$

Remove from the finite list $(\Gamma(y, u), \Pi(y, u)), u \geq 1$, all the pairs $(\Gamma(y, u), \Pi(y, u))$ such that: $\Gamma O_{<} \Gamma(y, u)$;

Insert the pair $\left(\Gamma, d^{*}(\Gamma)\right)$ in the list $(\Gamma(y, u), \Pi(y, u)), u \geq 1$, and adjust the values of the indices $u \geq 1$ which appear in this list in such a way that:

- the indices $u$ such that $\Gamma(y, u)$ is defined remain consecutive and ordered through increasing values of $\Pi(y, v)=d^{*}(\Gamma(y, v)$.

Theorem 4.1. The above algorithm STRAT-PATH2 computes a $k$ minimal strongly independent path family from $x_{0}$ to $y_{0}$ associated with $(R, O, L)$ and with the positive length function d, any time such a family exists.

Proof. We first check by induction the following loop invariant:

- Every time a pair $(x, i)$ is taken as pair (Pivot, Index-Pivot), the pathes $\Pi(x, j), j=1 \ldots i$, are elementary pathes and form a $i$ minimal strongly independent path family from $x_{0}$ to $x$ associated with $(R, O, L)$ and $d$. (I)

We may remark that, since $R$ and $O$ are stable under rigth side concatenation, since Nil $(O \vee R) \gamma$ for any $\gamma$ in $P^{*}(G)$, and since $d$ si positive, then any path $\Gamma(x, i)$ is elementary.

Let us suppose the assumption (I) to be wrong, and let us consider the pair $(x, i)$ which corresponds to the first time, during the execution of the algorithm, when (I) above becomes wrong.

That means that there exists some elementary path $\Gamma$ in $L$, from $x_{0}$ to $x$, such that $\Gamma O_{<} \Gamma(x, i)$ and also such that the pathes $\Gamma(x, j), j=1 \ldots i-1$, and $\Gamma$ are strongly $(O, R)$-independent.

We may suppose that $\Gamma$ is minimal for $O_{<}$with this property.

Then there exists some vertex $y \# x$ on $\Gamma$ and some index $j$ such that:

- the restriction $\Gamma_{x o, y}$ is the path $\Gamma(y, j)$;

- $z$ being the successor of $y$ on $\Gamma$, no index l exists such that $\Gamma_{x o, z}=\Gamma(z, l)$.

We may assume that, while taking into account the hypothesis on the $O_{<}$-minimality of $\Gamma$ we choosed $\Gamma$ in such a way that the number of vertices on the subpath $\Gamma_{y, x}$ is the smallest possible. 
Since $d$ is positive, since Nil $(O \vee R) \gamma$ and since $O$ and $R$ are stable under rigth side concatenation, we have $\Gamma(y, j) O_{<} \Gamma(x, i)$.

Because of hypothesis (H5), $\Gamma_{x o, z}$ is in $L$.

Since no index 1 exists such that $\Gamma_{x o, z}=\Gamma(z, l)$, there exists an index $u$ such that $\Gamma(z, u)\left(R_{=} \vee O_{<}\right) \Gamma_{x o, z}$.

Then, because $O$ and $R$ are stable under right side concatenation and because $d$ is positive, we have:

$$
\Gamma(z, u)+\Gamma_{z, x}\left(R_{=} \vee O_{<}\right) \Gamma
$$

The concatenation $\Gamma(z, u)+\Gamma_{z, x}$ may not be in $L$. But, because of hypothesis (H4), there exists a path $\Gamma^{\prime}=\Pi\left(\Gamma(z, u)+\Gamma_{z, x}\right)$ with origin $x_{0}$ and extremity $x$ which is in $L$ and which is such that:

$-\Gamma^{\prime} O_{<} \Gamma^{\prime}$ or $\Gamma^{\prime} R_{=} \Gamma$.

- $\Gamma^{\prime}$ is the concatenation of some prefix of $\Gamma(z, u)$ and of some path which does not contain more arcs than $\Gamma_{z, x}$.

Since $\Gamma(x, 1) \ldots \Gamma(x, i-1)$ and $\Gamma$ are strongly $(O, R)$-independent, so are $\Gamma(x, 1) \ldots \Gamma(x, i-1)$ and $\Gamma^{\prime}$.

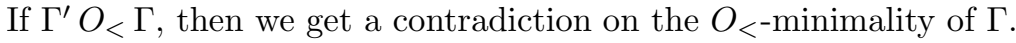

If $\Gamma^{\prime} R_{=} \Gamma$, then $\Gamma^{\prime}$ is the concatenation of a prefix $P$ of $\Gamma_{x o, z}$ and of some path $S$ which does not contain more arcs than $\Gamma_{z, x}$. Since $P$ can be written $\Gamma\left(z_{1}, i_{1}\right)$ for some vertex $z_{1}$ and some index $i_{1}$, it follows that we get a contradiction on the fact that $\Gamma$ and $y$ were chosen in order to make the number of vertices in $\Gamma_{y, x}$ minimal.

The fact that the invariant (I) holds implies that if the algorithm succeeds in marking the pair $\left(y_{0}, k\right)$ then what we get is really a $k$ minimal strongly independent path family from $x_{0}$ to $y_{0}$ associated with $(R, O, L)$ and $d$.

In case the algorithm fails in marking $\left(y_{0}, k\right)$, we need to check that no $k$ strongly independent path family from $x_{0}$ to $y_{0}$ associated with $(R, O, L)$ exists. In order to do this, we may proceed by exactly the same way as we just did before. We consider the largest index $i<k$ such that $\left(y_{0}, i\right)$ has been marked by the algorithm, we denote it by $i_{0}$, and we suppose the existence of some path $\Gamma$, minimal for $O_{<}$, such that the paths $\Gamma\left(y_{0}, 1\right), \ldots, \Gamma\left(y_{0}, i_{0}\right), \Gamma$ form a $\left(i_{0}+1\right)$ minimal strongly $(O-R)$ independent path family from $x_{0}$ to $y_{0}$. Once again, we consider an arc $[x, y]$ on $\Gamma$ such that:

- there exists $i$ such that the restriction $\Gamma_{x o, x}=\Gamma(x, i)$;

- there does not exist $j$ such that the restriction $\Gamma_{x o, y}$ is equal to some path $\Gamma(y, j)$.

We suppose that $\Gamma$ has also been chosen in such a way that the restriction $\Gamma_{y, y o}$ admits the less vertices possible. The fact that $\Gamma_{x o, y}$ is not equal to any 
path $\Gamma(y, j)$ means that there exists some index $u$ such that $\Gamma(y, u)$ exists and satisfies:

$$
-\Gamma(y, u) O_{<} \Gamma_{x o, y} \text { or } \Gamma(y, u) R_{=} \Gamma_{x o, y} .
$$

As we previously did, we notice that the concatenation of $\Gamma(y, u)$ and $\Gamma_{y, y o}$ may not be in $L$, but that its projection through $\Pi$ provides us with a contradiction with the hypothesises which we made about $\Gamma$.

\subsection{Complexity of the Algorithm STRATPATH2}

Our main problem comes here from the fact that before getting $\Gamma\left(y_{0}, k\right)$, STRATPATH2 will eventually compute many paths $\Gamma\left(x, k^{\prime}\right)$ with $k^{\prime}$ much larger than $k$. We cannot forecast a bound for $k^{\prime}$ and we notice that most of these intermediary results will not be really useful. So, we may, for a given parameter $I \geq k$, rewrite STRATPATH2 in such a way that it never keeps into memory any path $\Gamma\left(x, k^{\prime}\right)$ with $k^{\prime}>I$. Let us denote by STRATPATHBIS(I) the procedure obtained this way. Of course, STRATPATH2 and STRATPATHBIS(I) may yield different results.

So let us set the following definitions:

- Critical-Index $\left(G, x_{0}, y_{0}, \quad R, O, L, d\right)=$ the smallest value I such that STRATPATH2 and STRATPATHBIS(I) produce the same results from the input defined by the network $G=(X, E)$, the length function $d$, the origin/destination pair of vertices $x_{0}, y_{0}$, and the strategic triple $(R, O, L)$;

- $\operatorname{Index}(G, R, O, L)=\operatorname{Sup}$ Critical-Index $\left(G, x_{0}, y_{0}, R, O, L, d\right)$. $x_{0}, y_{0}$ in $X, d$ positive length function on $G$.

Let us also denote by $\tau(n)$ the complexity of testing that some path with $n$ arcs is in $L$, and by $\gamma(n)$ the complexity of comparing, for the $O$ and $R$ relationships, 2 paths of $P^{*}(G)$, with no more than $n$ arcs.

At any iteration of the main loop of STRATPATHBIS(I) some "For" loop is executed. This loop consists in scanning, for the current pair (Pivot, Index-Pivot) pair, the set of the vertices $y$ in $X$ such that [Pivot, $y$ ] is in $E$, and in updating the pathes $\Gamma(y, j), j=1 \ldots I$.

Thus any execution of the STRAT-PATHBIS(I) process contains at most $I$. $\sum_{x \text { in }} x d^{+} G(x)=I .|E|$ executions of this updating process, where $d^{+} G(x)$ denotes the outer degree in $G$ of the vertex $x$.

For such a vertex $y$ in $X$, such that [Pivot, $y$ ] is in $E$, this updating process may induce:

- checking the presence of some path in the set $L$;

- performing $|I|$ comparisons of 2 paths of $L$ for the $O$ and $R$ relationships.

We deduce that the complexity of STRAT-PATH(I) is no more than:

$$
O(I .|E| .(\tau(|X|)+I \cdot \gamma(|X|))
$$


We may state:

Proposition 1. The complexity of STRAT-PATH2 is bounded by

$$
O\left(\operatorname{INDEX}(G, O, R, L)^{2} \cdot(|E| \cdot(\tau(|X|)+\gamma(|X|)))\right) .
$$

Unfortunately, the fact is that $\operatorname{INDEX}(G, O, R, L)$ may be arbitrarily large.

Example. Let us consider some network $G=(X, E)$, given together with a distance function $d$ defined on the arc set $E$, with an alphabet $A$ and with some $A$-labelling of the arcs of $E$ as follows:

$-A=\left\{a_{0} \ldots a_{n}\right\}$

- $X=\{0,1 \ldots n,(0,1) \ldots(0, n)\}$

$-E=\{[i, i+1], i=0 \ldots n-1\}+\{[0,(0, i)], i=1 \ldots n,[(0, i), 1], i=1 \ldots n\}$;

- Any arc $[i, i+1]$ has length 1 and label $a_{i}$;

- Any arc $[0,(0, i)]$ has distance $1+i$ and label $a_{i}$;

- Any arc $[(0, i), 1], i=1 \ldots n$, has distance 1 and label $a_{0}$.

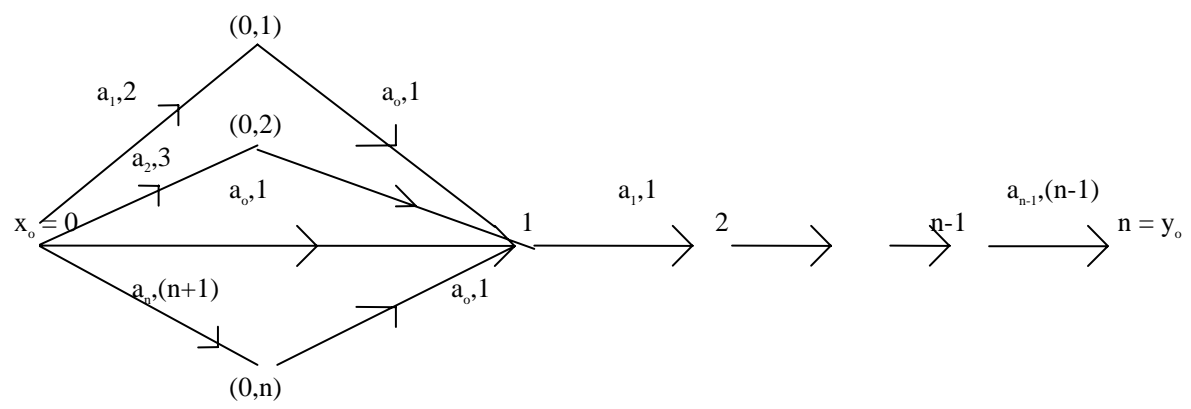

We suppose that: $x_{0}=0 ; y_{0}=n$.

We consider the strategic triple $(R, O, L)$ defined as follows:

- $L=P^{*}(G)=$ the set of all the possible pathes of $G$;

- $O=$ the trivial empty relation;

- $R: \gamma R \gamma^{\prime}$ iff any symbol which appears on $\gamma$ also appears in $\gamma^{\prime}$ and conversely.

Then, if $k=2$, a solution of Problem2 for the above input is given by the pathes:

$\left(x_{0}, 1, \ldots, n\right)$ and $\left(x_{0},(0, n), 1, \ldots, n\right)$ with respective lengthes $n$ and $2 n+1$. In order to compute it through the algorithm STRAT-PATH2, we need to compute the path $\Gamma(\mathbf{1}, \mathbf{n}+\mathbf{1})$.

Still, it is possible in many cases to bound the value of $\operatorname{Index}(R, O, L)$.

In order to see how to do it, let us consider some network $G=(X, E)$, some strategic triple $(R, O, L)$ and some length function d defined on $E$.

We call degree of $R$ in relation to $L$, denoted by $D(G, R, L)$, the largest integer s such that there exist $s+1$ pathes $\gamma, \gamma_{1} \ldots \gamma_{s}$ in $G$ which satisfy:

- the pathes $\gamma_{1} \ldots \gamma_{s}$ are pairwise $R$-independent and they share a same origin and a same extremity;

- the origin of $\gamma$ is the common extremity of the $\gamma_{i}, i=1 \ldots s$; 
- for any $i, j$ in $1 \ldots s, \Pi\left(\gamma_{i}+\gamma\right) R \Pi\left(\gamma_{j}+\gamma\right)$. (recall: $\Pi$ is the projection operator of the hypothesis (H4)).

Theorem 4.2. If $O$ is the empty partial ordering, then Index $(G, O, R, L)$ $\leq D(G, R, L)$.

Proof. Let us suppose the converse, that means let us suppose that some path $\Gamma(x, i)+\Gamma$ appears in the minimal strategy distinct $k$-path family from $x_{0}$ to $y_{0}$ computed by STRAT-PATH2, with $i>k . D(G, R, L)$.

Obviously we get a contradiction since the pathes $\Pi(\Gamma(x, i)+\Gamma), i=1 \ldots k$. $D(G, R, L)$ have their length no more than the length of $\Gamma(x, i)+\Gamma$, while it is possible to extract $k$ paths from these $k . D(G, R, L)$ pathes which are pairwise $R$-independent.

We may provide some examples of values $D(G, R, L)$ :

- Let us suppose that $G=(X, E)$, and $(R, O, L)$ are like in the example of Section 3.2. Then $D(G, R, L)=1$.

- Let us suppose that $G=(X, E)$ is such that any arc $e$ in $E$ is labelled with some symbol $a(e)$ in an alphabet $A$, and let us define $R$ and $L$ as follows:

$-L=P(G)^{*}$

- $\gamma R \gamma^{\prime}$ iff $\gamma$ globally involve the same symbols as $\gamma^{\prime}$ (independently of their multiplicity).

In such a case we have that $D(G, R, L)=2|A|$.

- Let us consider some network $G=(X, E)$ such that any arc $e$ in $E$ is labelled with some symbol $s(e)$ in an alphabet $A$, in such a way that: if $[x, y]$ and $[y, z]$ are two arcs which are endowed with a same label $s$, then there is an arc $[x, z]$ which is also labelled with $s$. Let us also suppose that:

- $L$ is the set of the alternated paths of $G$, that means the paths which do not contain any pair of consecutive arcs endowed with the same label;

- $\gamma R \gamma^{\prime}$ iff the $A$-words associated with $\gamma$ and $\gamma^{\prime}$ are the same.

In such a case we have that $D(G, R, L)=2$.

Unfortunately, it seems difficult to extend Theorem 2 to cases when the order relation $O$ is non empty.

However, we may remark (verification left to the reader) that:

- If the network $G=(X, E)$ and the strategic triple $(R, O, L)$ satisfy the following implication:

for any vertices $x, y, z$ in $G$, any paths $\gamma_{1}, \gamma_{2}, \gamma$ such that:

$\operatorname{or}(\gamma)=\operatorname{ext}\left(\gamma_{1}\right)=\operatorname{ext}\left(\gamma_{2}\right)=y$

$\operatorname{or}\left(\gamma_{1}\right)=\operatorname{or}\left(\gamma_{2}\right)=x ; \operatorname{ext}(\gamma)=z$;

$\gamma_{1}$ and $\gamma_{2}$ are both $O$-minimal in $P_{x, y}(G)$;

$\gamma_{1}+\gamma$ is minimal in $P_{x, z}(G)$;

then $\gamma_{2}+\gamma$ is also minimal in $P_{x, z}(G)$.

then $\operatorname{Index}(G, R, O, L)=D(G, R, L)$. 


\subsection{Algorithm Stratpath1 for the Problem 1}

The basic ideas for solving Problem 1 are the same as those which we just previously presented. They may be summarized as follows:

\section{Algorithm STRAT-PATH1}

Input: The network $G=(X, E)$, the origin/destination pair $\left(x_{0}, y_{0}\right)$, the strategic $\overline{\text { triple }}(R, O, L)$ and the integer $k$.

Output: Failure or a $k$ minimal independent path family from $x_{0}$ to $y_{0}$ associated with the strategic triple $(R, O, L)$.

$\Gamma\left(x_{0}, 0\right):=$ Trivial path reduced to the vertex $x_{0}$;

Not Stop; No pair $(x, i)$ is marked;

While Not Stop do

Set (Pivot, Index-Pivot) $:=$ some pair $(x, i)$ such that $\Gamma(x, i)$ exists,

is not marked, and is minimal for the relation $O$ with these properties;

Mark (Pivot, Index-Pivot);

If (Pivot, Index-Pivot) does not exist then Stop (failure)

\section{Else}

If $($ Pivot, Index-Pivot $)=\left(y_{0}, k\right)$ then Stop (Success: the $\Gamma\left(y_{0}, i\right)$,

$i=1 \ldots k$, yield the result)

Else

For any arc $[x, y]$, such that $\Gamma(x, i)+\{[x, y]\}$ is in $L$ and is not $R$-equivalent to any path $\Gamma(y, j), j$ in $\mathrm{N}$ do

Set $\Gamma:=\Gamma(x, i)+\{[x, y]\}$;

Let $k(y)$ be the largest index $j$ such that $\Gamma(y, j)$ exists;

Let $u_{0}$ be the largest index $u$ such that: Not $((\Gamma(x, i)+$ $\{[x, y]\}) O \Gamma(y, u))$;

For $j:=k(y)$ downto $u_{0}+1$ do $\Gamma(x, j+1):=\Gamma(x, j)$;

$\Gamma\left(y, u_{0}+1\right):=\Gamma$;

Theorem 4.3. The above algorithm STRAT-PATH1 effectively computes a $k$ minimal independent path family from $x_{0}$ to $y_{0}$ associated with the strategic triple $(R, O, L)$, every time that such a family exists.

We leave the proof of this result to the reader, since it is very close to the proof of our next result.

\section{Numerical EXPERIMENTS}

We present here two classes of experiments, both related to the example of Section 3.2. The first one tests the quantity $\operatorname{Index}(\mathbf{O}, \mathbf{R}, \mathbf{L})$ which was introduced in the previous Section 4.2. The second one aims at comparing the results produced by the STRATPATH2 algorithm with the results produced by a stochastic approach of the same problem of the search for independent efficient routing strategies in a network. 
So we consider some network $G=(X, E)$, some integer $k, 2$ vertices $x_{0}$ and $y_{0}$, and we suppose that every arc $e$ in $E$ is endowed with 2 positive coefficients $\alpha(e)$ and $\lambda(e)$ and with some symbol $U(e)$, belonging to some set $U$ of symbolic variables.

Then, to every path $\gamma=\left\{e_{0} \ldots e_{n}\right\}$ in $G$, corresponds some symbolic $U$ expression:

$$
\Lambda^{*}(\gamma)=\sum_{i=0 \ldots n} \alpha\left(e_{i}\right)+\lambda\left(e_{i}\right) \cdot U\left(e_{i}\right)
$$

The relations $R$ and $O$ are defined by:

- $\gamma R \gamma^{\prime}$ iff the symbolic $U$-expressions $\Lambda^{*}(\gamma)$ and $\Lambda^{*}\left(\gamma^{\prime}\right)$ are the same;

- $\gamma O \gamma^{\prime}$ iff $\Lambda^{*}(\gamma)<\Lambda^{*}\left(\gamma^{\prime}\right)$ whatever be the feasible value taken by $U$.

$L$ is defined as the set of all the possible pathes on $G$.

In order to test the quantity Index $(O, R, L)$ on such an input, we use:

- two classes of networks with 100 vertices:

- "dense" networks: the presence of an arc in the arc set is determined through random sorting, with a probability $\mathrm{p}$ which may takes values $0.1,0.2,0.4$

- "sparse" networks: the outer degree of any vertex may not to exceed some number $q=4,6$ or 8 .

- a symbolic variable set $U$ with 5 or 10 symbols;

$-k=4,6$ or 8 , and values for the coefficients $\lambda(e)$ and $\alpha(e)$ randomly sorted between 1 and 5 ;

- origin/destination pairs $x_{0}, y_{0}$ randomly sorted, while distinguishing "close" pairs $\left(x_{0}\right.$ and $y_{0}$ may be connected by a path with no more than 5 arcs) from the other pairs.

For any test, we compute the value Critical-Index $=$ Critical-Index $(G, O, R, L$, $\left.x_{0}, y_{0}\right)$ defined in Section 4.2.

Then for any sequence $S$ of identically parameted experiments, we get the following quantities:

- Ind-Min $=$ Inf Critical-Index, taken for all the experiments in $S$;

- Ind-Max = Sup Critical-Index, taken for all the experiments in $S$;

- Ind-Mean = Mean value of Critical-Index, taken for all the experiments in $S$;

- Double $=$ Proportion of the experiments which provided a value of CriticalIndex larger than $2 k$.

The results which we get may be summarized according to the following arrays:

We notice that:

- in most of the cases (more than $80 \%$ of the cases), Critical-Index is no more than $2 k$;

- increases in the "density" of $G$, or in the distance between origin $x_{0}$ and destination $y_{0}$, or in the coefficient $k$, tend to deteriorate the value of CriticalIndex. 


\begin{tabular}{|c|c|c|c|c|}
\hline & Ind-Min & Ind-Max & Ind-Mean & Double \\
\hline \multicolumn{5}{|c|}{ "Dense" and "Close" } \\
\hline$k=4$ & 4 & 10 & 5.4 & $48 / 50(96 \%)$ \\
\hline \multirow[t]{2}{*}{$k=8$} & 8 & 27 & 13.5 & $42 / 50(84 \%)$ \\
\hline & Ind-Min & Ind-Max & Ind-Mean & Double \\
\hline \multicolumn{5}{|c|}{ "Dense" and "Far" } \\
\hline$k=4$ & 4 & 13 & 6.5 & $44 / 50(88 \%)$ \\
\hline \multirow[t]{2}{*}{$k=8$} & 8 & 34 & 16 & $32 / 50(64 \%)$ \\
\hline & Ind-Min & Ind-Max & Ind-Mean & Double \\
\hline \multicolumn{5}{|c|}{ "Sparse" and "Close" } \\
\hline$k=4$ & 4 & 8 & 5.2 & $48 / 50(96 \%)$ \\
\hline \multirow[t]{2}{*}{$k=8$} & 8 & 22 & 10.4 & $44 / 50(88 \%)$ \\
\hline & Ind-Min & Ind-Max & Ind-Mean & Double \\
\hline \multicolumn{5}{|c|}{ "Sparse" and "Far" } \\
\hline$k=4$ & 4 & 10 & 6.2 & $45 / 50(90 \%)$ \\
\hline$k=8$ & 8 & 28 & 12.6 & $39 / 50(78 \%)$ \\
\hline
\end{tabular}

Our second class of experiments must be related to our initial discussion of the semantics of the problem (Sections 1 and 2). Let us recall that we introduced this model of "strongly independent path family", in order to help us in computing paths which are at the same time efficient, and independent in relation to the possible variations of some state vector. But in the context of the above specific example, we could have tried to reach this goal through an other approach: we could have proceeded by randomly generating a sequence of values for the vector $U$, and by looking for a shortest path in $G$ for the resulting positive length function. Namely, we could have applied the following GENER-PATH Procedure, which is a kind of Monte-Carlo algorithm:

\section{GENER-PATH(N, k):}

Input: A network $G=(X, E)$, such that any arc $e$ in $E$ is endowed with some formal linear affine expression $t(e, U)=\alpha(e)+\lambda(e) \cdot u(e)$, where $\alpha(e)$ and $\lambda((e)$ are positive coefficients and where $u(e)$ is some symbolic variable, and 2 vertices $x_{0}, y_{0}$ in $X$;

Output: Some family of strongly $(O, R)$-independent path;

\section{For $i:=1$ to $N$ do}

Randomly generate a value for the "speed" vector $U$, according to some distribution $\sigma$, with mean value in 1 ;

Compute a shortest path $\gamma_{i}$ from $x_{0}$ to $y_{0}$ in $G$, associated with the distance function which to any arc $e$ in $E$ makes correspond the value of $t(e, U)$; 
Extract from the paths computed this way, a set $S$ of strategies $(R$ equivalence classes) ordered according to the number of representents of these strategies among the pathes $\gamma_{i}, i=1 \ldots N$;

For $i=1$ to $k$, select some path which represents the $i$-th element of $S$.

Obviously, the paths generated this way form a strongly $(O, R)$-independent path family. This family may not be minimal. Conversely, given some path $\gamma$ in a minimal independent path family, there may not exist any value of $U$ such that $\gamma$ is a shortest path for the length function $t(., U)$. For instance one may easily see that a strongly $(O, R)$-independent 3 path family may be made with 3 paths $\gamma_{1}, \gamma_{2}, \gamma_{3}$ such that for any value of $U$, the following inequality holds:

$$
\Lambda^{*}\left(\gamma_{1}, U\right)+\Lambda^{*}\left(\gamma_{2}, U\right)<2 . \Lambda^{*}\left(\gamma_{3}, U\right)
$$

and then check that such a relation forbids $\gamma_{3}$ from being a shortest path for any well-chosen value of $U$.

Still, one intuitively feels that both procedures GENER-PATH and STRATPATH2 aim at the same goal. In order to compare them, we use the same tests as in the previous part (with $N=100$ ), while looking, for any test, to the number SHARE of elements shared by the lists produced by GENER-PATH and by STRAT- PATH2. Then, for any sequence $\mathrm{S}$ of identically parameted experiments, we compute the following quantities:

- Share-Min = Inf SHARE, taken for all the experiments in $S$;

- Share-Max = Sup SHARE, taken for all the experiments in $S$;

- Share-Mean = Mean value of SHARE, taken for all the experiments in $S$;

- Share $=$ Proportion of the experiments which provided a value of SHARE equal to $k$.

Then the following arrays summarize our results:

Comment. Though the procedures STRAPATH2 and GENER-PATH do not formally deal with the same problems, we see that they yield similar results. So, it becomes natural to compare their respective running times. Though we did not implement our algorithms in order to optimize their running times, we could check that if the number of vertices of the network $G$ is large enough, then the running time of STRATPATH2 increases like the quantity $k .|U|^{2}$ (the case $k=1,|U|=1$ corresponds to an execution of Dijkstra's algorithm), while the running time of GENER-PATH evolves like the parameter $N$. When $k=5,|U|=5, N=100$, and when we deal with the same "sparse" networks as above, then the running times of STRATPATH2 and GENER-PATH are close to each other. But when $k=5,|U|=10, N=100$, and when we deal with the same "dense" networks as above, then the ratio between the respective running times of STRATPATH2 and GENER-PATH is close to 7, which makes the Monte-Carlo procedure GENERPATH appear more efficient than STRATPATH2. 


\begin{tabular}{lllll}
\hline & Share-Min & Share-Max & Share-Mean & Share \\
\hline "Dense" and "Close" & & & & \\
$k=4$ & 2 & 4 & 3.2 & $15 / 50$ \\
$k=8$ & 5 & 8 & 6.5 & $8 / 50$ \\
& Share-Min & Share-Max & Share-Mean & Share \\
"Dense" and "Far" & & & & \\
$k=4$ & 2 & 4 & 2.9 & $12 / 50$ \\
$k=8$ & 5 & 8 & 5.8 & $7 / 50$ \\
& Share-Min & Share-Max & Share-Mean & Share \\
"Sparse" and "Close" & & & & \\
$k=4$ & 3 & 4 & 3.5 & $25 / 50$ \\
$k=8$ & 5 & 8 & 6.8 & $10 / 50$ \\
& Share-Min & Share-Max & Share-Mean & Share \\
"Sparse" and "Close" & & & & \\
$k=4$ & 2 & 4 & 3 & $20 / 50$ \\
$k=8$ & 5 & 8 & 6 & $8 / 50$ \\
\hline
\end{tabular}

\section{Conclusion And Perspectives}

We just introduced here a new independence concept adapted to the search for collections of routing strategies in a network. This led us to deal, for a given number $k$, with the problem of finding a $k$ minimal independent path family connecting two given vertices in a network. In order to solve this problem, we designed the algorithm STRATPATH2 which may be viewed as an extension of DIJKSTRA's algorithm. We studied the theoretical complexity of this algorithm, and performed experimentations in order to test its behaviour. We also compared the results produced by this algorithm with the results obtained through application of some empirical "Monte-Carlo" process.

It would be interesting now to think more about potential applications of this model. At first, our motivation came from practical routing problems related to the control of Transportation or Telecommunications systems subject to state variations. More recently, we worked (partnership with CERTU- LYON and SNCF) on problems related to the strategical design of such systems. Those problems involve the design of some infrastructure (communication links, vehicle flows) which will support, for a given set of origin/destination pairs, the routing of various kinds of objects (passengers, informations, goods.) between these origin/destination pairs. The associated models are obtained by considering the communication infrastructure as a master variable, and every origin/destination routing flow as an auxiliary variable. They may be handled through local optimization procedures. 
Thus, in order to avoid dealing with the routing subproblems related to every origin/destination pair every time the master "infrastructure" variable is updated, we may think into predetermining, for every origin/destination pair, some path family computed in such a way that, whatever be the final infrastructure, one among these pathes is likely to provide us with a convenient routing decision associated with this pair.

Under this prospect, we studied a specific academic problem which consists in computing routes and frequencies for a public transportation system in order to make him maximize time-elastic demands. This gave us an opportunity to adapt this idea by using path families which are minimal independent path families in the sense of this paper, and to compare the induced results with those produced by a more classical approach. As part of a continuation of this research, we plan providing more details about this experimentation in the context of a future presentation.

\section{REFERENCES}

[1] F. Bendali and A. Quilliot, Réseaux stochastiques. RAIRO Oper. Res. 24 (1990) 167-190.

[2] T.H. Cormen, C.H. Leiserson and R.L. Rivest, Introduction to algorithms. MIT Press, Cambridge, Mass (1980).

[3] E. Dijkstra, A note with two problems in connection with graphs. Numer. Math. I (1959) 269-271.

[4] R. Dionne and M. Florian, Exact and approximate algorithms for optimal network design. Network 9 (1979) 37-59.

[5] A. Farley, Minimum broadcast networks. Networks 10 (1980) 59-70.

[6] M. Florian, No linear cost models in transportation analysis. Math. Programming Study 26 (1986) 167-196.

[7] P. Fraigniaud and E. Lazard, Methods and problems of communication in usual networks. Discrete Appl. Math. 53 (1994) 79-133.

[8] M. Gondran and M. Minoux, Graphes et algorithmes. Ed. Eyrolles (1979).

[9] R.M. Karp and J.B. Orlin, Parametric shortest path algorithms with application to cyclic staffing. Discrete Appl. Math. 3 (1981) 37-45.

[10] J. Lauriere, Intelligence Artificielle. Eyrolles (1987).

[11] E. Lawler, A procedure for computing the $k$ best solutions to discrete optimization problems and its application to the shortest path problem. Management Sci. 18 (1972) 401-405.

[12] E. Minieka, Optimization algorithms for networks and graphs. Marcel Dekker Inc. (1978).

[13] M. Minoux, Network synthesis and optimum network design problems: Models, solution methods and applications". Network 19 (1989) 313-360.

[14] N. Nilsson, Problem solving methods in A.I. Mac Graw Hill (1971).

[15] A. Quilliot, A retraction problem in graph theory. Discrete Math. 54 (1985) 61-71.

[16] A. Quilliot, Algorithmes de cheminements pour des réseaux d'actions à effets non déterministes. Math. Appl. 12 (1991) 25-44.

[17] M. Sakarovitch, Chemins, flots, ordonnancements dans les réseaux. Hermann, Paris (1984).

[18] M. Sakarovitch, The $k$ shortest routes and $k$-shortest chains in a graph. Report ORC, Operation Research Center, University of California, Berkeley (1966) 66-32. 
[19] D.R. Shier, On algorithms for finding the $k$ shortest pathes in a network. Networks 9 (1979) 195-214.

[20] J. Wardrop, Some theoretical aspects of road traffic research. Proc. Inst. Civil Engrg. II (1952) 325-378.

[21] B. Yaged, Minimum cost routing for dynamic network models. Network 3 (1973) 315-331.

to access this journal online: www.edpsciences.org 